\title{
Ganho de peso de novilhas em pastagem nativa da Serra do Sudeste do RS submetida ao controle de plantas indesejáveis e intensidades de pastejo
}

\author{
Heifers weight gain on Serra do Sudeste native pasture in RS, submitted to undesirable plants control \\ and grazing intensities
}

\author{
Leonardo Araripe Crancio ${ }^{1}$ Paulo César de Faccio Carvalho ${ }^{2}$ Carlos Nabinger $^{3}$ \\ Jamir Luis Silva da Silva ${ }^{4}$ Rogério Jaworski dos Santos ${ }^{5}$ Davi Teixeira dos Santos ${ }^{5}$ \\ Luiz Giovani de Pellegrini ${ }^{5}$
}

\section{RESUMO}

Este trabalho teve como objetivo avaliar o efeito de métodos de controle de espécies indesejáveis e de intensidades de pastejo sobre o desempenho animal em pastagem nativa da Serra do Sudeste do Rio Grande do Sul. Foram utilizados os métodos de controle: roçada de primavera $(R P)$, roçada de outono (RO), controle químico (Q) e um tratamento testemunha (T), todos submetidos a dois níveis de oferta de forragem, médio (8\%) e alto (14\%), expressos em $\mathrm{kg}$ de matéria seca diariamente ofertada para cada $100 \mathrm{~kg}$ de peso vivo animal. $O$ delineamento experimental empregado foi o de blocos completamente casualizados, num arranjo fatorial com duas repetições. A análise estatística não demonstrou interação entre os fatores estudados, bem como existência de diferença entre os métodos de controle de espécies indesejáveis para as variáveis de produção primária e secundária $(P>0,1)$. Não houve diferença de ganho médio diário entre os níveis de oferta de forragem estudados $(P>0,1)$. Animais submetidos à alta oferta de forragem apresentaram menor perda de peso por unidade de área $(\mathrm{P}<0,1)$.

Palavras-chave: controle químico, oferta de forragem, roçada.

\section{ABSTRACT}

This work aimed to evaluate the effect of different undesirable plants controlling methods and grazing intensities on animal production in native pastures. The treatments were no control $(T)$, spring clipping $(P)$, autumn clipping $(O)$ and chemical control $(Q)$, under two levels of forage allowance, medium (8\%) and high (14\%), expressed in $\mathrm{kg}$ of dry matter offered daily per each $100 \mathrm{~kg}$ of liveweight. The experimental design was a completely randomized block arranged in a factorial with two repetitions. Statistical analysis showed no interaction among treatments nor differences among control methods for variables concerning primary and secondary production $(P>0.1)$. Animal performance in both forage allowances was not different $(P>0.1)$. High forage allowances increased animal performance per unit area $(P<0.1)$.

Key words: chemical control, herbage allowance, clipping.

\section{INTRODUÇÃO}

A exploração de gado de corte no Rio Grande do Sul tem como principal substrato o campo nativo. Uma de suas principais características é a heterogeneidade temporal e espacial, sendo esta última relacionada às características químicas e físicas de solo, à precipitação, à temperatura, ao relevo e a altitude. Já a heterogeneidade temporal pode ser ilustrada pela curva de produção anual de forragem. Esta produção varia em função da composição botânica da pastagem, ou seja, da predominância de espécies de rota metabólica $\mathrm{C}_{3}$ ou $\mathrm{C}_{4}$, que determinam a dinâmica da produção de forragem ao longo das estações do ano e definem o balanço anual de produção de forragem. A sazonalidade na produção de forragem é uma das características deste ecossistema; porém, parte dos

\footnotetext{
${ }^{1}$ Programa de Pós-graduação em Zootecnia, Universidade Federal do Rio Grande do Sul (UFRGS), Porto Alegre, RS, Brasil. E-mail: lcrancio@yahoo.com.br.

${ }^{2}$ Departamento de Plantas Forrageiras e Agrometeorologia, UFRGS, Av. Bento Gonçalves, 7712, 91501-970, Porto Alegre, RS, Brasil. E-mail: paulocfc@ufrgs.br. Autor para correspodência.

${ }^{3}$ Departamento de Plantas Forrageiras e Agrometeorologia, UFRGS, Porto Alegre, RS, Brasil. E-mail: nabinger@ufrgs.br.

${ }^{4}$ Departamento de Plantas Forrageiras e Agrometeorologia, UFRGS, Porto Alegre, RS, Brasil. E-mail: jamirlss@ufrgs.br.

${ }^{5}$ Programa de Pós-graduação em Zootecnia, UFRGS, Porto Alegre, RS, Brasil.
} 
produtores não ajusta o manejo dos animais a esta situação. Como conseqüência, tem-se no inverno um desempenho animal inferior quando comparado àquele do período favorável, atribuído principalmente à baixa quantidade e qualidade da forragem consumida.

Desta forma, o uso de lotações fixas ou a não-adequação da demanda animal à estacionalidade produtiva da pastagem contribui para os modestos índices da produção pecuária no Rio Grande do Sul (elevada idade de abate de machos, elevada idade do primeiro acasalamento de fêmeas e baixo índice de natalidade). Esta situação é incoerente se for considerada a diversidade florística da pastagem, onde há, segundo BOLDRINI (1997), aproximadamente 450 espécies de gramíneas e 150 de leguminosas.

A exploração do ambiente de forma que seja possível compatibilizar a produção animal com a preservação do meio ambiente é uma forma racional de utilização do recurso forrageiro nativo e pode ser conseguida através do emprego de uma "pressão de pastejo ótima” (NABINGER, 1998).

Portanto, fica evidenciado que este substrato forrageiro só é improdutivo quando manejado de forma inadequada, o que traz, num primeiro instante, um baixo desempenho animal por limitar a ingestão de forragem por indivíduo. Num segundo momento, a limitação de ingestão de forragem pode se dar por diminuição da área útil pastoril, já que o manejo inadequado ocasiona alteração da composição botânica da pastagem, favorecendo o desenvolvimento de plantas indesejáveis.

No contexto da produção animal, planta indesejável nativa é aquela que, mesmo fazendo parte do ecossistema, diminui a produção de espécies de interesse forrageiro por competir por recursos nutricionais, água e luz e por diminuir a área de pastejo, ou ainda, pela interação entre esses dois fatores. Também caracteriza-se por não integrar de forma constante a dieta dos animais, além de poder apresentar efeitos tóxicos. A presença dessas espécies implica a diminuição da capacidade de suporte da pastagem, sendo que algumas ainda podem danificar o couro ou a lã dos animais. Existem várias espécies nativas indesejáveis no Rio Grande do Sul, porém as mais freqüentes são a carqueja (Baccharis trimera Less.), o alecrim do campo (Vernonia nudiflora Less.), o caraguatá (Eryngium horridum Malme), o mio-mio (Baccharis coridifolia D.C.) e a chirca (Eupatorium buniifolium Hook). Quanto aos métodos de controle existentes, podem ser citados os métodos mecânicos, químicos, e biológicos. Porém, inúmeras vezes a integração de métodos se faz necessária para atingir uma maior eficiência de controle, além de um efeito mais duradouro, como pode ser visto nos trabalhos de GONZAGA et al. (1998) e CARÁMBULA et al. (1995).

Os trabalhos existentes na área de controle de plantas indesejáveis consideram o método e as épocas de controle, mas raramente estudam a interação destes com a intensidade de pastejo, assim como seus reflexos sobre a produção animal. Esta pesquisa teve como objetivo avaliar a produção animal em pastagem nativa da Serra do Sudeste do RS sob diferentes métodos de controle de plantas indesejáveis e intensidades de pastejo.

\section{MATERIAL E MÉTODOS}

O experimento foi conduzido na Fazenda do Angico, no município de Cachoeira do Sul, na região fisiográfica da Serra do Sudeste (RS). As avaliações tiveram início em 15 de março de 2003 e término em 8 de julho de 2003, compreendendo 116 dias de ocupação e utilização da pastagem. Aárea experimental foi dividida em 16 potreiros (unidades experimentais), com área média de 2,63 ha, totalizando 42,1 hectares, além de uma área anexa disponível para os animais reguladores da oferta de forragem. O experimento foi primeiramente estabelecido por FONTOURA JUNIOR (2003) em 2002 e, posteriormente, permaneceu diferido de outubro de 2002 a março de 2003.

A composição botânica do estrato superior da pastagem era constituída por carqueja (Baccharis trimera), capim caninha (Andropogon lateralis), barba de bode (Aristida laevis) e alecrim (Vernonia nudiflora), e, no estrato inferior, sobressaíam entre as gramíneas a grama forquilha (Paspalum notatum) e a grama tapete (Axonopus affinis) e, entre as leguminosas, Arachis burkartii, Stylosanthes leiocarpa e o pega-pega (Desmodium incanum). A composição florística completa da área está descrita em FONTOURA JUNIOR (2003).

Os tratamentos aos quais a pastagem e os animais experimentais foram submetidos constituíramse de diferentes métodos de controle de espécies indesejáveis: testemunha $(\mathrm{T})$, roçada mecânica de primavera (RP) e de outono (RO), controle químico (Q); e dois níveis de oferta de forragem: médio (8\%) e alto (14\%), expressos em kg de matéria seca diariamente ofertada para cada $100 \mathrm{~kg}$ de peso vivo animal. Para o controle químico, empregou-se um herbicida que tem como princípio ativo 2,4 D + Picloram. As roçadas de primavera foram realizadas em outubro de 2001 e em janeiro de 2003, enquanto que as roçadas de outono foram realizadas na segunda quinzena de maio de 2002 e na primeira quinzena de junho de 2003. O método de pastejo empregado foi o contínuo com lotação variável. 
Foram realizadas pesagens dos animais nos dias 25 de março, 3 de maio e 8 de julho de 2003, com o objetivo de ajustar a carga animal conforme a oferta de forragem pretendida. Para este ajuste, utilizou-se a técnica "put and take”, mantendo no mínimo três animais (testes) por unidade experimental para obtenção de adequada precisão nas medidas quantitativas da pastagem e um número de animais reguladores segundo a oferta pretendida (BRANSBY, 1989). Utilizou-se novilhas sem padrão racial definido, com idade média de 18 meses e peso médio de $257,9 \mathrm{~kg}$ e desvio padrão de $28 \mathrm{~kg}$.

A taxa de acúmulo de matéria seca (TAD) foi obtida através da metodologia descrita por MORAES et al. (1990), utilizando-se três gaiolas de exclusão do pastejo por unidade experimental, realocadas a cada 28 dias. A matéria seca obtida dentro da gaiola (DGi), subtraída da matéria seca da amostra de fora da gaiola no período anterior (FGi-1), dividida pelo número de dias entre as amostragens (n), ou seja, $\mathrm{TAD}=(\mathrm{DGi}-\mathrm{Fgi}-1) / \mathrm{n}$, forneceu a taxa de acúmulo de forragem em $\mathrm{kg}$ de MS/ha/dia. A massa de forragem foi avaliada por meio do método do rendimento visual comparativo (HAYDOCK \& SHAW, 1975), desconsiderando-se as espécies indesejáveis. Em cada data de amostragem, estimou-se, para o tratamento testemunha e para os demais tratamentos, 80 e 60 pontos por unidade experimental, respectivamente. Para gerar as equações de regressão e validar as estimativas visuais, foram estabelecidos dez pontos abrangendo toda a variação de biomassa da pastagem, que, uma vez cortados e secos, foram utilizados juntamente com os pontos fora de gaiola, provenientes do triplo emparelhamento. Os padrões do tratamento testemunha eram específicos devido à diferença na estrutura da pastagem.

As pesagens que determinaram o início e o final do período experimental foram precedidas de jejum de 16 horas. Obteve-se o ganho médio diário (GMD) pela diferença entre o peso final e inicial dos animais testes, dividido pelo número de dias do período. A carga animal foi obtida por meio do somatório do peso médio dos animais testes e reguladores de cada potreiro, dividido pela área de cada um, e expressa em kg de PV $\mathrm{ha}^{-1}$. Esta variável teve seu valor ponderado devido aos sub-períodos terem diferença quanto ao número de dias. A divisão deste valor pelo peso médio dos animais testes forneceu a taxa de lotação animal ( $\left(\mathrm{an} \mathrm{ha}^{-1}\right)$. O valor para a variável animais.dia ha ${ }^{-1}$ foi obtido multiplicandose a taxa de lotação pelo número de dias do período de avaliação. A produção de quilogramas de peso vivo por hectare (GPV) foi obtida pelo produto do GMD pelo animal.dia ha-1 ${ }^{-1}$ O controle sanitário constituiu-se de vacinação contra aftosa, brucelose, carbúnculo, vermífugo (a cada 45 dias) e controle periódico de ectoparasitas.

O delineamento experimental foi o de blocos completamente casualizados, utilizando-se como critério de bloqueamento as diferenças de cobertura das indesejáveis e o relevo da área. Os tratamentos constituíram um esquema fatorial $4 \times 2$, sendo quatro métodos de controle e duas ofertas de forragem, com duas repetições. No bloco 1, o herbicida foi aplicado de forma total (tratorizado), na dose de $4 \mathrm{~L} \mathrm{ha}^{-1}$. Já no bloco 2, foi utilizado o método de aplicação localizada, com aparelho costal, na concentração de 1,5\%. Em ambos os blocos, a aplicação ocorreu na primeira quinzena de abril de 2002.

Os dados referentes às variáveis GMD, GPV e TAD receberam transformação logarítmica de base dez para ajuste do coeficiente de variação. Os dados foram submetidos à análise de variância e teste F pelo procedimento GLM do pacote estatístico SAS (SAS, 2001). Quando detectadas diferenças ao nível de $10 \%$ entre os métodos de controle, as médias foram comparadas pelo teste Tukey, também a $10 \%$ de significância.

\section{RESULTADOS E DISCUSSÃO}

Na medida em que não houve interação $(\mathrm{P}>0,1)$ entre os níveis de oferta e os métodos de controle sobre todas as variáveis associadas ao desempenho animal, como se observa na tabela 1 , a discussão desses fatores foi feita de forma independente. De acordo com a tabela 2, as variáveis associadas ao desempenho animal não apresentaram diferença $(\mathrm{P}>0,1)$ entre os métodos de controle utilizados. Todavia, a menor perda de peso registrada para o tratamento utilizando roçada de primavera pode ser relevante para o planejamento reprodutivo do rebanho por tratarem-se de fêmeas. Provavelmente, o tratamento roçada de primavera proporcionaria aos animais a entrada no período de primavera com um peso mais próximo daquele necessário para o acasalamento.

Para o cálculo da oferta real de forragem, foi utilizado um valor médio de taxa de acúmulo diário (TAD) para todos os tratamentos devido à grande variabilidade dos valores encontrados para essa variável, fato este que pode ser atribuído às condições climáticas comuns desta época do ano, neste ambiente, associado a erros decorrentes da própria metodologia. Os valores utilizados foram - 4,3 e - 1,9kg de matéria seca ha ${ }^{-1}$ dia $^{-1}$ para o primeiro (15/03 a 01/05) e segundo (01/05 a 21/06) subperíodos, respectivamente. Porém, para a análise estatística dessa variável, utilizaram-se 
Tabela 1 - Resumo da análise de variância das variáveis carga animal (kg de PV ha ${ }^{-1}$ ), lotação (an. ha ${ }^{-1}$ ), número de animais-dia por hectare ( an. dia $^{-1} \mathrm{ha}^{-1}$ ), ganho médio diário (GMD, $\mathrm{kg} \mathrm{an}^{-1}$ dia $^{-1}$ ), ganho de peso vivo por área ( $\mathrm{kg}$ de PV ha ${ }^{-1}$ ), massa de forragem (MF, $\mathrm{kg}$ de MS ha ${ }^{-1}$ ), taxa de acúmulo diário (TAD, kg de MS ha ${ }^{-1}$ dia $^{-1}$ ), e oferta real de forragem ( $\mathrm{kg}$ de MS 100kg-1 de PV) para níveis de oferta de forragem (OF), métodos de controle (MC) de espécies indesejáveis e interação entre níveis de oferta e métodos de controle (OF*MC).

\begin{tabular}{|c|c|c|c|c|c|}
\hline \multirow[b]{2}{*}{ Variável } & \multicolumn{3}{|c|}{ Probabilidade } & \multirow{2}{*}{$\mathrm{CV}(\%)$} & \multirow{2}{*}{$\mathrm{R}^{2}(\%)$} \\
\hline & OF & MC & $\mathrm{OF}^{*} \mathrm{MC}$ & & \\
\hline Carga, kg de PV ha ${ }^{-1}$ & 0,0162 & 0,3969 & 0,6819 & 20,26 & 70,49 \\
\hline Lotação, an. ha ${ }^{-1}$ & 0,0317 & 0,4427 & 0,6309 & 28,81 & 65,19 \\
\hline Animais.dia ha ${ }^{-1}$ & 0,0320 & 0,4441 & 0,6333 & 23,87 & 65,06 \\
\hline GMD, kg an ${ }^{-1} \mathrm{dia}^{-1}$ & 0,1806 & 0,5976 & 0,2918 & 6,80 & 68,11 \\
\hline GPV, kg de PV ha ${ }^{-1}$ & 0,0225 & 0,7091 & 0,4292 & 14,27 & 67,44 \\
\hline MF, kg de MS ha ${ }^{-1}$ & 0,0465 & 0,1509 & 0,3646 & 16,46 & 70,71 \\
\hline TAD, kg de MS ha ${ }^{-1}$ dia $^{-1}$ & 0,9657 & 0,4595 & 0,5434 & 24,95 & 46,11 \\
\hline Of real, kg de MS100 kg de PV ${ }^{-1}$ & 0,0414 & 0,9013 & 0,9111 & 29,40 & 57,01 \\
\hline
\end{tabular}

$\mathrm{CV}=$ Coeficiente de varição.

$\mathrm{R}^{2}=$ coeficiente de determinação.

os valores absolutos, próprios de cada unidade experimental. Já que não foi detectada diferença $(\mathrm{P}>0,1)$ entre os níveis de oferta de forragem, propôs-se a forma do referido cálculo para a oferta real de forragem. Os resultados apresentados na tabela 2 poderiam, em princípio, sugerir que a escolha pela utilização de determinado método devesse estar relacionada apenas com o componente econômico. No entanto, parece mais coerente recomendar o estudo de possíveis impactos ecológicos de um ou outro método de controle, visando a uma conclusão mais consistente em torno desta temática.

Na tabela 3, observam-se os valores médios de produção segundo os níveis de oferta de forragem. Apesar de as ofertas reais de forragem terem sido superiores às ofertas pretendidas inicialmente, houve diferença entre os dois níveis $(\mathrm{P}<0,1)$, contemplando a proposta inicial do trabalho. Os valores efetivos de oferta de forragem foram de 11,6 e 16,8\% para os tratamentos média e alta oferta, respectivamente. As

Tabela 2 - Carga animal (kg de PV ha ${ }^{-1}$ ), lotação $\left(a n . ~ h a^{-1}\right.$ ), número de animais-dia por hectare (an.dia ${ }^{-1} \mathrm{ha}^{-1}$ ), ganho médio diário (GMD, kg $\mathrm{an}^{-1} \mathrm{dia}^{-1}$ ), ganho de peso vivo por área ( $\mathrm{kg}$ de PV ha ${ }^{-1}$ ), massa de forragem (MF, kg de MS ha ${ }^{-1}$ ), taxa de acúmulo diário (TAD, $\mathrm{kg}$ de MS ha ${ }^{-1} \mathrm{dia}^{-1}$ ), oferta real de forragem ( $\mathrm{kg}$ de MS $100 \mathrm{~kg}^{-1} \mathrm{de}$ PV) e diferença mínima significativa (DMS), segundo os tratamentos de métodos de controle (média de 4 unidades experimentais).

\begin{tabular}{|c|c|c|c|c|c|}
\hline \multirow[b]{2}{*}{ Variáveis } & \multicolumn{4}{|c|}{ Métodos de Controle } & \multirow{2}{*}{ DMS } \\
\hline & Testemunha (T) & Roçada de Primavera (RP) & Roçada de Outono (RO) & Químico (Q) & \\
\hline Carga, kg de PV ha ${ }^{-1}$ & 532,58 & 475,65 & 550,06 & 618,54 & 261,71 \\
\hline Lotação, an. ha ${ }^{-1}$ & 2,07 & 1,97 & 2,13 & 2,57 & 1,02 \\
\hline Animais.dia ${ }^{-1} \mathrm{ha}^{-1}$ & 240,79 & 229,45 & 247,22 & 298,2 & 119,14 \\
\hline GMD, $\mathrm{kg} \mathrm{an}^{-1} \mathrm{dia}^{-1}$ & $-0,220$ & $-0,146$ & $-0,190$ & $-0,182$ & 0,146 \\
\hline GPV, kg de PV ha-1 & $-52,05$ & $-37,74$ & $-45,11$ & $-52,84$ & 40,15 \\
\hline MF, kg de MS ha $^{-1}$ & 3500,3 & 2971,8 & 3473,4 & 4070,7 & 1134,1 \\
\hline TAD, kg de MS $\mathrm{ha}^{-1} \mathrm{dia}^{-1}$ & $-2,65$ & $-13,37$ & $-5,91$ & 5,65 & 30,47 \\
\hline OF real, kg de MS $100 \mathrm{~kg}^{-1}$ de PV & 13,7 & 13,7 & 13,6 & 15,5 & 8,18 \\
\hline
\end{tabular}




\begin{tabular}{|c|c|c|c|}
\hline \multirow[b]{3}{*}{ Variáveis } & \multicolumn{2}{|c|}{ Oferta de forragem } & \multirow{3}{*}{ DMS } \\
\hline & Média & Alta & \\
\hline & (8kg de MS $100 \mathrm{~kg}^{-1}$ de PV) & (14kg de MS $100 \mathrm{~kg}^{-1}$ de PV) & \\
\hline Carga, kg de PV ha ${ }^{-1}$ & $631 *$ & 458 & 104,45 \\
\hline Lotação, an. ha ${ }^{-1}$ & $2,5 *$ & 1,8 & 0,4938 \\
\hline Animal.dia ${ }^{-1} \mathrm{ha}^{-1}$ & $294,4 *$ & 213,4 & 57,421 \\
\hline GMD, $\mathrm{kg} \mathrm{an}^{-1} \mathrm{dia}^{-1}$ & $-0,212$ & $-0,157$ & 0,0705 \\
\hline GPV, kg de PV ha ${ }^{-1}$ & $-61,8$ & $-32, *$ & 19,353 \\
\hline MF, kg de MS ha ${ }^{-1}$ & 3155,8 & $3852,3 *$ & 546,61 \\
\hline TAD, kg de MS ha-1 $\mathrm{dia}^{-1}$ & $-3,54$ & $-3,48$ & 14,405 \\
\hline Of real, $\mathrm{kg}$ de MS $100 \mathrm{~kg}^{-1}$ de PV & 11,6 & $16,8 *$ & 3,9448 \\
\hline
\end{tabular}

*Significativo $(\mathrm{P}<0,1)$ pelo teste $\mathrm{F}$.

diferenças $(\mathrm{P}<0,1)$ observadas para as variáveis carga animal, lotação, animais.dia ha-1 e massa de forragem (MF) foram conseqüências das diferenças entre os níveis de oferta de forragem. A carga animal no tratamento com média e alta oferta de forragem foi de 631 e $458 \mathrm{~kg}$ de peso vivo ha ${ }^{-1}$, respectivamente. Estes valores são superiores aos obtidos por SETELICH (1994), de 494 e $429 \mathrm{~kg}$ de peso vivo ha- ${ }^{-1}$ respectivamente para as ofertas 12 e $16 \%$, no período de verão-outono, que, juntamente com os valores das demais ofertas utilizadas (4 e 8\%), geraram uma resposta linear negativa em função dos níveis de oferta estudados. Por sua vez, CORRÊAA (1993) obteve uma resposta quadrática para o período verão-outono, sendo seus valores de 460 , 378 e $430 \mathrm{~kg}$ de peso vivo ha $^{-1}$ para os níveis 8, 12 e $16 \%$ de oferta de forragem. Já SOARES (2002), trabalhando com estes mesmos níveis de oferta, não encontrou diferença $(\mathrm{P}>0,05)$ entre os valores de carga animal para o período de outono, registrando valores de 351 e $486 \mathrm{~kg}$ de peso vivo ha-1 para as respectivas ofertas de 12 e $16 \%$. As cargas animais obtidas no presente estudo foram mais altas em função dos elevados valores de massa de forragem em relação aos anteriores, mantendo assim a relação necessária entre massa de forragem e carga animal quando do ajuste da oferta em cada tratamento.

Quanto à expressão da carga animal na forma de animais.dia/ha, esta apresentou diferença $(\mathrm{P}<0,1)$ entre os níveis médio e alto de oferta de forragem. Esta variável permite a comparação com outros trabalhos, já que leva em consideração a duração do período experimental. SETELICH (1994) encontrou valores de 207 e 183 animais.dia ha ${ }^{-1}$ utilizando animais com dois anos de idade, no período de verão-outono, para as ofertas de forragem 12 e 16\%, respectivamente; valores que foram inferiores aos obtidos neste experimento.

A massa de forragem (MF) é conseqüência dos tratamentos de oferta aplicados e apresentou diferença entre os níveis estudados $(\mathrm{P}<0,1)$, sendo o tratamento com $14 \%$ de oferta de forragem o de maior massa de forragem, confirmando que diferentes intensidades de pastejo definem a existência de diferentes quantidades de forragem. Os elevados valores da massa de forragem neste experimento podem ser justificados pelo extenso período de tempo em que a área ficou diferida. A relação linear positiva entre oferta de forragem e massa de forragem foi verificada em vários trabalhos (ESCOSTEGUY, 1990; MOOJEN, 1991; CORRÊA, 1993; SETELICH, 1994 e SOARES, 2002). A massa de forragem no presente estudo teve valores superiores aos encontrados pelos autores citados, sendo que, destes, somente MOOJEN (1991), entre maio e julho, obteve valor próximo (3792kg/ha) para o nível elevado de oferta de forragem em pastagem nativa do RS.

O GMD não diferiu ( $\mathrm{P}>0,1)$, apesar dos valores de -0,212 e -0,157kg verificados respectivamente para os níveis de oferta 10 e $14 \%$ do PV. Esse resultado pode ser explicado, em parte, pelo fato de que as ofertas reais corresponderam a valores próximos do ponto de máximo GMD em pastagem nativa, como pode ser visto na curva de ganho de peso por níveis de oferta de forragem desenvolvida por MARASCHIN (1998). Nesta curva, o GMD máximo ocorre na oferta de 13,5\% e, quanto mais próximo deste ponto, menos esta variável é afetada pela variação da oferta de forragem. No presente estudo, as ofertas reais foram da ordem de 
$11,6 \%$ e 16,8\%, ou seja, estão em pontos eqüidistantes com valores abaixo e acima da oferta que proporciona maior GMD. Os valores nos dois tratamentos foram negativos, com perda média total de 24 e $18 \mathrm{~kg}_{\text {animal }}{ }^{-1}$ em 114 dias de pastejo, respectivamente, para as ofertas de forragem média e alta. Tal desempenho deve estar relacionado, sobretudo, com o aspecto qualitativo da forragem. Deve ser destacado que o período em questão não é favorável à produção de forragem e ao desempenho de animais a pasto. Na mesma área experimental, FONTOURA JUNIOR (2003) também não encontrou diferença entre os dois níveis de oferta de forragem estudados ( 8 e $14 \%$ ), sendo que no tratamento $14 \%$ o ganho foi praticamente nulo e no tratamento $8 \%$ houve pequena perda de peso. Um baixo desempenho animal também foi verificado por MOOJEN (1991), no qual o GMD no período outono-inverno foi de $-0,173 \mathrm{~kg} \mathrm{an}^{-1} \mathrm{dia}^{-1}$ para o tratamento $12 \%$ e de,$- 007 \mathrm{~kg}$ $\mathrm{an}^{-1} \mathrm{dia}^{-1}$ para o tratamento $16 \%$ de oferta de forragem. No entanto, SOARES (2002), no período de outono, obteve 0,217 e $0,115 \mathrm{~kg} \mathrm{an}^{-1} \mathrm{dia}^{-1}$ para as ofertas de forragem 12 e $16 \%$, respectivamente. Já no inverno, os valores foram de $-0,083$ e $-0,069 \mathrm{~kg} \mathrm{an}^{-1} \mathrm{dia}^{-1}$ para as mesmas ofertas de forragem. Registros de baixo desempenho animal em pastagem natural durante a estação fria do ano são de há muito tempo conhecidos no Rio Grande do Sul. MOOJEN (1991), citando GROSSMAN \& MOHRDIECK (1956), relata que neste período, em Uruguaiana, São Gabriel e Vacaria, houve perdas de 11, 28 e 62 \% do ganho obtido na estação favorável. Isto demonstra a variabilidade das condições ambientais no Estado, dentro deste período do ano.

Em relação ao desempenho por área, houve diferença $(P<0,1)$ entre os níveis de oferta estudados, sendo que, no menor nível, a perda de peso foi de aproximadamente duas vezes superior à perda do maior nível. A diferença constatada entre os níveis de oferta é atribuída, principalmente, à diferença entre as taxas de lotação. Entretanto, a pequena diferença numérica observada para GMD também contribuiu para a diferença do GPV ha-1 entre os níveis de oferta. Valores de 19,2 e 3,3kg de peso vivo ha-1, no período outonal, foram observados por SOARES (2002) para as ofertas de 12 e 16\%, respectivamente. Enquanto isso, no inverno, as perdas variaram de $-3,7$ a $-11,5 \mathrm{~kg}$ de peso vivo ha ${ }^{-1}$ para as mesmas ofertas de forragem. Para FONTOURA JUNIOR (2003), no período outonoinverno, a variável GPV/ha diferiu entre ofertas, apresentando um ganho de $1,9 \mathrm{~kg} /$ ha no tratamento $14 \%$ e perda de $13,6 \mathrm{~kg} \mathrm{ha}^{-1}$ no tratamento $8 \%$.

Quanto à taxa de acúmulo, de acordo com a tabela 3, os valores obtidos foram de -3,54 e de -3,48kg de MS ha-1 para os tratamentos médio e alto nível de oferta de forragem, não apresentando diferença $(\mathrm{P}>0,1)$. Este comportamento das taxas de acúmulo também foi relatado por MOLAN (2004), que, avaliando Brachiaria brizantha por meio de quatro alturas de dossel (10, 20, 30 e $40 \mathrm{~cm}$ ), obteve taxas de acúmulo decrescentes no inverno, apresentando como valor médio dos tratamentos $-13,5 \mathrm{~kg}$ de MS ha ${ }^{-1} \mathrm{dia}^{-1}$ para uma massa de forragem média de $1.2340 \mathrm{~kg}_{\text {de }} \mathrm{MS} \mathrm{ha}^{-1}$. Erros na estimativa do acúmulo de forragem podem estar relacionados ao método utilizado para quantificar esta variável, assim como ao intervalo de tempo entre as amostragens nas áreas excluídas do pastejo (BORTOLO et al., 2001). Isto ocorre devido à variação no índice de área foliar (IAF) dentro e fora da gaiola, podendo-se inferir que áreas protegidas do pastejo e com baixos resíduos de matéria seca passariam rapidamente de um baixo IAF para um IAF ótimo, no qual ocorre a máxima taxa de acúmulo de matéria seca, alcançando em seguida o IAF teto. Em situações de valores altos de massa de forragem, as condições no interior da gaiola já estariam próximas do IAF teto e a taxa de crescimento começaria a diminuir. Isto ocorre principalmente em função do sombreamento imposto pelas folhas do estrato superior sobre aquelas do estrato inferior, as quais acabariam se transformando em material senescente e morto.

\section{CONCLUSÕES}

Para as condições de pastagem nativa da Serra do Sudeste do RS, os métodos de controle de plantas indesejáveis roçada de primavera e roçada de outono ou controle químico não alteraram o ganho de peso de novilhas no período de outono-inverno, em relação a áreas sem qualquer tipo de controle.

Mesmo com alta oferta de forragem, não foi possível manter o peso de novilhas em pastagem nativa da Serra do Sudeste no período de outono-inverno, com perda de peso por animal semelhante à obtida com oferta de forragem moderada. Esta última representou melhor utilização da área neste período, com resultados superiores para as variáveis relacionadas à capacidade de suporte.

\section{AGRADECIMENTOS}

Os autores agradecem à Empresa Agropecuária Cerro Coroado pela disponibilização dos animais e da área experimental; à Dow Agrosciences, pelo apoio financeiro, e à Coordenação de Aperfeiçoamento de Pessoal de Nível Superior (CAPES) e ao Conselho Nacional de Desenvolvimento Científico e Tecnológico (CNPq), pela concessão de bolsas. 


\section{REFERÊNCIAS}

BOLDRINI, I.I. Campos do Rio Grande do Sul: caracterização fisionômica e problemática ocupacional. Porto Alegre: Universidade Federal do Rio Grande do Sul, 1997. 39p. (Boletim do Instituto de Biociências, 56).

BORTOLO, M. et al. Avaliação de uma pastagem de coastcross1 (Cynodon dactylon (L.) Pers) sob diferentes níveis de matéria seca residual. Revista Brasileira de Zootecnia,Viçosa, v.30, n.3, p.627-635, 2001.

BRANSBY, D.I. Compromisses in the design and conduct of grazing experiments. In: MARTEN, G.C. (Ed). Grazing research design, methodology and analysis. Alabama: Crop Science Society of America and American Society of Agronomy, 1989. p.53-67.

CARÁMBULA, M. et al. Control de cardilla. Montevideo: INIA, 1995. 9p. (Boletim técnico, 57).

CORRÊA, F.L. Produção e qualidade de uma pastagem nativa do Rio Grande do Sul. 1993. 165f. Dissertação (Mestrado em Zootecnia) - Programa de Pós-graduação em Zootecnia, Faculdade de Agronomia, Universidade Federal do Rio Grande do Sul.

ESCOSTEGUY, C.M.D. Avaliação agronômica de uma pastagem natural sob níveis de pressão de pastejo. 1990. 231f. Dissertação (Mestrado em Zootecnia) - Programa de Pós-graduação em Zootecnia, Faculdade de Agronomia, Universidade Federal do Rio Grande do Sul.

FONTOURA JUNIOR, J.A.S. Controle de plantas indesejáveis em pastagem nativa da serra do sudeste do RS, sob a influência da intensidade de pastejo associada a métodos químicos e físicos. 2003. 77f. Dissertação (Mestrado em Zootecnia) - Programa de Pós-graduação em Zootecnia, Faculdade de Agronomia, Universidade Federal do Rio Grande do Sul.

GONZAGA, S.S. et al. Utilização de herbicidas no controle de plantas indesejáveis em pastagem natural. In: REUNIÃO DO GRUPO TÉCNICO EM FORRAGEIRAS DO CONE SUL ZONA CAMPOS, 17., 1998, Lages. Anais... Lages: EPAGRI/ UDESC, 1998 . 156p. p.142.

GROSSMAN, J.; MOHRDIECK, K.H. Experimentação forrageira do RS. In: RIO GRANDE DO SUL, SECRETARIA DA AGRICULTURA. DIRETORIA DA PRODUÇÃO ANIMAL. Histórico da diretoria da produção animal. Porto Alegre: Secretaria da Agricultura do Rio Grande do Sul, 1956. p.115122 .
HAYDOCK, K.P.; SHAW, N.H. The comparative yield method for estimating dry matter yield of pasture. Australian Journal Agronomy and Animal Husbandry, Melbourne, v.15, p.6670, 1975.

MARASCHIN, G.E. Utilização, manejo e produtividade das pastagens nativas da região sul do Brasil. In: CICLO DE PALESTRAS EM PRODUÇÃO E MANEJO DE BOVINOS DE CORTE, 3., 1998, Canoas. Anais... Canoas: ULBRA, 1998. 105p. 107p. p.29-39.

MOLAN, L.K. Estrutura do dossel, interceptação luminosa e acúmulo de forragem em pastos de CapimMarandu submetidos a alturas de pastejo por meio de lotação contínua. 2004. 159f. Dissertação (Mestrado em Agronomia - Ciência Animal e Pastagens) - ESALQ, Universidade de São Paulo.

MOOJEN, E.L. Dinâmica e potencial produtivo de uma pastagem nativa do Rio Grande do Sul submetida a pressões de pastejo, épocas de diferimento e níveis de adubação. 1991. 172f. Tese (Doutorado em Zootecnia) Programa de Pós-graduação em Zootecnia, Faculdade de Agronomia, Universidade Federal do Rio Grande do Sul.

MORAES, A. et al. Comparação de métodos de taxa de crescimento em uma pastagem submetida a diferentes pressões de pastejo. In: REUNIÃO ANUAL DA SOCIEDADE BRASILEIRA DE ZOOTECNIA, 27., 1990, Campinas. Anais... Campinas: SBZ, 1990. p332.

NABINGER, C. Princípios de Manejo e Produtividade de Pastagens. In: CICLO DE PALESTRAS EM PRODUÇÃO E MANEJO DE BOVINOS DE CORTE, 3., Canoas. Anais... Canoas: ULBRA, 1998. 107p. p.54-107.

SAS INSTITUTE. System for information. Versão 6. 11, Cary, 2001. CD Rom.

SETELICH, E.A. Potencial produtivo de uma pastagem natural do Rio Grande do Sul, submetida a distintas ofertas de MS. 1994. 169f. Dissertação (Mestrado em Zootecnia) - Programa de Pós-graduação em Zootecnia, Faculdade de Agronomia, Universidade Federal do Rio Grande do Sul.

SOARES, A.B. Efeito da alteração da oferta de matéria seca de uma pastagem natural sobre a produção animal e a dinâmica da vegetação. 2002. 180f. Tese (Doutorado em Zootecnia) - Programa de Pós-Graduação em Zootecnia, Faculdade de Agronomia, Universidade Federal do Rio Grande do Sul. 